\title{
Commentary on the KDIGO 2021 Clinical Practice Guideline for the Management of Blood Pressure in CKD
}

\author{
Johannes F. E. Mann ${ }^{1}$ (D) Tara I. Chang ${ }^{2}$ - William C. Cushman ${ }^{3}$. Susan L. Furth ${ }^{4}$. Joachim H. Ix ${ }^{5}$ Fan Fan Hou ${ }^{6}$. \\ Gregory A. Knoll ${ }^{7}$. Paul Muntner ${ }^{8}$. Roberto Pecoits-Filho ${ }^{9,10}$ • Mark J. Sarnak ${ }^{11}$ • Charles R. V. Tomson ${ }^{12}$.

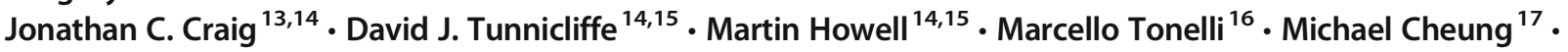 \\ ${\text { Amy } \text { Earley }^{17} \text {. Alfred K. Cheung }}^{18}$
}

Accepted: 30 April 2021 / Published online: 16 August 2021

(C) The Author(s), under exclusive licence to Springer Science+Business Media, LLC, part of Springer Nature 2021

\begin{abstract}
Purpose of Review To summarize and explain the new guideline on blood pressure (BP) management in chronic kidney disease (CKD) published by Kidney Disease: Improving Global Outcomes (KDIGO), an independent global nonprofit organization which develops and implements evidence-based clinical practice guidelines in kidney disease. KDIGO issued its first clinical practice guideline for the Management of Blood Pressure (BP) in Chronic Kidney Disease (CKD) for patients not receiving dialysis in 2012 and now updated the guideline in 2021.

Recent Findings Recommendations in this update were developed based on systematic literature reviews and appraisal of the quality of the evidence and strength of recommendation following the "Grading of Recommendations Assessment, Development and Evaluation" (GRADE) approach. The updated guideline includes five chapters covering BP measurement techniques, lifestyle interventions for lowering BP, and management of BP in three target populations, namely adults (with and without diabetes), kidney transplant recipients, and children. A dedicated chapter on BP measurement emphasizing standardized preparation and measurement protocols for office BP measurement is a new addition, following protocols used in large randomized trials of BP targets with pivotal clinical outcomes.

Summary Based on the available evidence, and in particular in the CKD subgroup of the SPRINT trial, the 2021 guideline suggests a systolic BP target of $<120 \mathrm{~mm} \mathrm{Hg}$, based on standardized measurements, for most individuals with CKD not receiving dialysis, with the exception of kidney transplant recipients and children. This recommendation is strictly contingent on the measurement of BP using standardized office readings and not routine office readings.
\end{abstract}

This article is part of the Topical Collection on Hypertension

Johannes F. E. Mann

Johannes.mann@kms.mhn.de

Alfred K. Cheung

Alfred.Cheung@hsc.utah.edu

1 KfH Kidney Center, University Hospital, Friedrich Alexander University, Erlangen, Nuremberg, Germany

2 Stanford University, Palo Alto, CA, USA

3 University of Tennessee Health Science Center, Memphis, TN, USA

4 Perelman School of Medicine, University of Pennsylvania and The Children's Hospital of Philadelphia, Philadelphia, PA, USA

5 Nanfang Hospital, Southern Medical University, Guangzhou, Guangdong, China

6 University of California San Diego and Veterans Affairs San Diego Healthcare System, San Diego, CA, USA

7 The Ottawa Hospital, Ottawa Hospital Research Institute, Ottawa, Canada
8 University of Alabama at Birmingham, Birmingham, AL, USA

9 Arbor Research Collaborative for Health, Ann Arbor, MI, USA

10 Pontifical Catholic University of Paraná, Curitiba, PR, Brazil

11 Tufts University, Boston, MA, USA

12 Freeman Hospital, Newcastle upon Tyne Hospitals NHS Trust, Newcastle upon Tyne, UK

13 College of Medicine and Public Health, Flinders University, Adelaide, South Australia, Australia

14 Cochrane Kidney and Transplant, Sydney, New South Wales, Australia

15 Sydney School of Public Health, The University of Sydney, Sydney, New South Wales, Australia

16 University of Calgary, Calgary, Alberta, Canada

17 KDIGO, Brussels, Belgium

18 University of Utah Health Center, Salt Lake City, UT, USA 
Keywords Albuminuria - Angiotensin-converting enzyme inhibitor · Angiotensin II receptor blocker - Blood pressure measurement $\cdot$ Blood pressure targets $\cdot$ Children $\cdot$ Chronic kidney disease $\cdot$ Dietary sodium $\cdot$ Kidney transplant recipient . Standardized office blood pressure

\section{Introduction}

The Kidney Disease: Improving Global Outcomes (KDIGO) is a global nonprofit organization that develops and implements evidence-based clinical practice guidelines in kidney disease. In 2012, KDIGO published a guideline for Management of Blood Pressure (BP) in Chronic Kidney Disease (CKD) for patients not receiving dialysis. Since then, the Systolic Blood Pressure Intervention Trial (SPRINT), with a dedicated CKD subgroup, sponsored by the National Institutes of Health was published. Several national and international organizations have since revised their guidelines and alluded to BP management in CKD but considerations of BP management with CKD were not comprehensively addressed. Such guidelines include those by the American College of Cardiology (ACC)/American Heart Association (AHA), European Society of Cardiology (ESC)/European Society of Hypertension (ESH), Hypertension Canada, and National Heart Foundation of Australia. KDIGO commissioned a dedicated evidence review and revision of the guidelines due to availability of new informative data in this area.

The current article summarizes the first three of the five chapters of the KDIGO 2021 guideline for Management of BP in CKD not receiving dialysis. Our focus will be the two respective chapters on BP measurement and on BP targets. These chapters are closely related to each other as the systolic blood pressure (SBP) target of $<120 \mathrm{~mm}$ $\mathrm{Hg}$ (Chapter 3) is fully contingent on standardized office BP measurement (Chapter 1). We will also briefly present the major features in Chapter 2 related to lifestyle management, but not BP in kidney transplant recipients (Chapter 4) and BP in children (Chapter 5). Readers are referred to the main guideline for these areas $[1 \bullet \bullet$.

\section{How Should Blood Pressure Be Measured? (Chapter 1)}

In the new KDIGO 2021 BP guideline, there is a chapter dedicated to proper BP measurement. In particular, the new guideline recommends standardized office BP measurement in preference to routine office BP measurement for the management of high BP in adults. The Work Group chooses to offer a strong recommendation (Grade $1 B$ ) based on GRADE standards and moderate-quality evidence. The major rationale behind the recommendation is that all large randomized controlled trials (RCTs) conducted with the main aim of examining target BP used standardized, not routine, office BP measurements. The same applies to RCTs testing specific drugs to lower BP. Thus, the Work Group did not choose standardized office BP on the basis of its precision or other aspects but simply its use to examine BP targets in RCTs.

Routine measurements of BP are by definition not standardized and likely executed in vastly differing ways from one clinic to the next, and often from one patient to the next. Thus, routine BP measurements yield highly variable values with unpredictable relationship to standardized BP.

Procedures to follow for standardized office BP are presented in Table 1, adapted from the 2017 ACC/AHA BP guideline [2]. Routine office BP refers to measurements performed without following the procedures outlined in Table 1 and is often called casual office BP. Because of strong evidence that the relationship between routine office BP and standardized office BP is highly variable in an individual, it is not possible to apply a correction factor to translate a routine $B P$ value to standardized BP in a given individual. In a wellexecuted study in CKD patients, mean SBP with standardized technique was $12.7 \mathrm{~mm} \mathrm{Hg}$ lower than with routine technique with wide variability from approximately +20 to $-45 \mathrm{~mm} \mathrm{Hg}$ $[3 \cdot \bullet]$. Therefore, whenever diagnostic or therapeutic decisions on BP management are taken, they should be based on standardized office BP (Table 2).

The guideline further provides practice points that favor the use of an automated oscillometric BP device over a manual device for standardized office BP measurement. Of note, standardization includes the procedures outlined in Table 1 but not the type of equipment. In our experience, the use of automated office BP (AOBP), attended or unattended, may free healthcare workers to undertake other duties. Both SPRINT and ACCORD used AOBP, attended or unattended, and both trials have major impact on the present guideline [4].

Out-of-office BP measurements were not used in major outcome trials that examined target BPs or antihypertensive drugs, except in children with CKD. Such out-of-office measurements include home BP monitoring (HBPM) and 24-h ambulatory BP monitoring (ABPM). HBPM is easier to implement than ABPM and may be used when a clinic visit is impractical (e.g., during the coronavirus 2019 pandemic). The guideline issues a weak recommendation for the use of out-ofoffice BP measurements as a complement to standardized office $\mathrm{BP}$, partly because there are no large RCTs comparing the effects of lower versus higher BP goals on clinical outcomes that used out-of-office measurements to guide the BP 
Table 1 Checklist for standardized office blood pressure measurement
1 Properly prepare the patient

2 Use proper technique for BP measurements

3 Take the proper measurements needed for diagnosis and treatments of elevated BP

4 Properly document accurate BP readings

5 Average the readings

6 Provide BP readings to patient
1 Have the patient relax, sitting in a chair (feet on floor, back supported) for $>5 \mathrm{~min}$

2 The patient should avoid caffeine, exercise, and smoking for at least $30 \mathrm{~min}$ before measurement

3 Ensure patient has emptied his/her bladder

4 Neither the patient nor the observer should talk during the rest period or during the measurement

5 Remove all clothing covering in the location of cuff placement

6 Measurements made while the patient is sitting or lying on an examining table do not fulfill these criteria

1 Use a BP measurement device that has been validated, and ensure that the device is calibrated periodically

2 Support the patient's arm (e.g., resting on a desk)

3 Position the middle of the cuff on the patient's upper arm at the level of the right atrium (the midpoint of the sternum)

4 Use the correct cuff size, such that the bladder encircles $80 \%$ of the arm, and note if a larger- or smaller-than-normal cuff size is used

5 Either the stethoscope diaphragm or bell may be used for auscultatory readings

1 At the first visit, record BP in both arms. Use the arm that gives the higher reading for subsequent readings

2 Separate repeated measurements by 1-2 min

3 For auscultatory determinations, use a palpated estimate of radial pulse obliteration pressure to estimate SBP. Inflate the cuff $20-30 \mathrm{~mm} \mathrm{Hg}$ above this level for an auscultatory determination of the BP level

4 For auscultatory readings, deflate the cuff pressure $2 \mathrm{~mm} \mathrm{Hg}$ per second, and listen for Korotkoff sounds

1 Record SBP and DBP. If using the auscultatory technique, record SBP and DBP as onset of the first Korotkoff sound and disappearance of all Korotkoff sounds, respectively, using the nearest even number

2 Note the time of most recent BP medication taken before measurements

Use an average of $\geq 2$ readings obtained on $\geq 2$ occassions to estimate the individual's level of BP

Provide patients with the SBP/DBP readings verbally and in writing

$B P$, blood pressure; $D B P$, diastolic blood pressure; $S B P$, systolic blood pressure. (Reprinted from: Whelton PK, et al. J Am Coll Cardiol 2018; 71: e127-e248, with permission from Elsevier) [2]

intervention. However, there are observational studies which show a stronger association of out-of-office BP measurements with cardiovascular and kidney outcomes than standardized office BP measurements in the general population and CKD population. HBPM may also promote adherence to antihypertensive interventions especially used in conjunction with cointerventions such as telemonitoring or pharmacist care [5•].

\section{What Is the Role of Lifestyle Management for Lowering BP in CKD Patients Not Receiving Dialysis? (Chapter 2)}

This chapter focuses on the antihypertensive effects of lifestyle in CKD, but it does not address broader health issues, such as metabolic effects of physical exercise, 
Table 2 Key guidance from the 2021 KDIGO Clinical Practice Guideline for BP Management in CKD
Chapter 1: Blood pressure measurement
Recommendation We recommend standardized office BP measurement in preference to routine office BP measurement for the management of high BP in adults $(1 B)$.
1.1
We suggest that out-of-office BP measurements with ambulatory BP monitoring (ABPM) or home BP monitoring (HBPM) be used to complement standardized office BP readings for the management of high $\mathrm{BP}(2 B)$.

Chapter 2: Lifestyle interventions for lowering blood pressure in patients with CKD not receiving dialysis
Recommendation We suggest targeting a sodium intake $<2$ g of sodium per day (or $<90$ mmol of sodium 2.1.1

Recommendation 2.2.1 per day, or $<5 \mathrm{~g}$ of sodium chloride per day) in patients with high BP and CKD (2C).

We suggest that patients with high BP and CKD be advised to undertake moderate-intensity physical activity for a cumulative duration of at least $150 \mathrm{~min}$ per week, or to a level compatible with their cardiovascular and physical tolerance $(2 C)$.

Chapter 3: Blood pressure management in patients with $\mathrm{CKD}$, with or without diabetes, not receiving dialysis

Recommendation We suggest that adults with high BP and CKD be treated with a target systolic blood 3.1.1 pressure (SBP) of $<120 \mathrm{~mm} \mathrm{Hg}$, when tolerated, using standardize office BP measurement $(2 B)$.

Recommendation We recommend starting (RASi) therapy (ACEi or ARB) for people with high BP, CKD, 3.2.1 and severely increased albuminuria (G1-G4, A3) without diabetes $(1 B)$.

Recommendation We suggest starting RASi (ACEi or ARB) for people with high $\mathrm{BP}, \mathrm{CKD}$, and moderately 3.2.2 increased albuminuria $(\mathrm{G} 1-\mathrm{G} 4, \mathrm{~A} 2)$ without diabetes $(2 \mathrm{C})$.

Recommendation We recommend starting RASi (ACEi or ARB) for people with high BP, CKD, and 3.2 .3 moderately-to-severely increased albuminuria (G1-G4, A2 and A3) with diabetes (1B).

Recommendation We recommend avoiding any combination of ACEi, ARB, and direct renin inhibitor 3.3.1 (DRI) therapy in patients with CKD, with or without diabetes $(1 B)$

Chapter 4: Blood pressure management in kidney transplant recipients (CKD G1T-G5T)

Practice Point 4.1 Treat adult kidney transplant recipients with high BP to a target $\mathrm{BP}$ of $<130 \mathrm{~mm} \mathrm{Hg}$ systolic and $<80 \mathrm{~mm} \mathrm{Hg}$ diastolic using standardized office BP measurements (see Recommendation 1.1)

Recommendation We recommend that a dihydropyridine calcium channel blocker or an ARB be used as the $4.1 \quad$ first-line antihypertensive agent in adult kidney transplant recipients $(1 C)$.

Chapter 5: Blood pressure management in children with CKD

Recommendation We suggest that in children with CKD, 24-h mean arterial pressure by ABPM should be 5.1 lowered to $\leq 50$ th percentile for age, sex, and height $(2 \mathrm{C})$.

$A B P M$, ambulatory BP monitoring; $A C E i$, angiotensin-converting enzyme inhibitor; $A R B$, angiotensin II receptor blocker; $B P$, blood pressure; $C K D$, chronic kidney disease; $R A S i$, renin-angiotensin system inhibitor

smoking, obesity, or alcohol consumption, beyond the effects on BP.

Dietary salt restriction reduces elevated BP in the short term in the general population and in the CKD population when salt intake is high. The guidelines suggest that CKD patients with high $\mathrm{BP}$ and a high salt consumption reduce salt intake to $<2 \mathrm{~g}$ sodium per day (Table 2 ). The recommendation is weak (Grade $2 C$ ) because direct evidence in the CKD population is weak. The systematic review conducted for this guideline found no RCT data evaluating the effects of dietary salt reduction on cardiovascular disease, kidney failure, or mortality in the CKD population. In addition, even in BP trials in the general population, few participants actually adhere to a diet with $<2 \mathrm{~g}$ /day of sodium in the long term. Recent meta-analyses of RCTs in non-CKD populations, however, demonstrate a graded benefit in both BP and cardiovascular disease risk reduction with reductions in salt intake, that is from high to moderate intake, even if $<2 \mathrm{~g} /$ day is not achieved. While data on specific targets of salt intake in CKD populations with high BP are not firmly established, and as KDIGO guidelines are designed to serve an international audience, the Work Group adopted the recommended dietary salt intake target for the general population from the World Health Organization (WHO) [6]. Finally, this recommendation is also consistent with the recently published KDIGO 2020 Guideline for Diabetes Management in CKD [7]. 
The guideline mentions problems with salt restriction, namely in CKD with salt-wasting nephropathy and the use of potassium-containing salt substitutes. Diets recommended to lower BP are often rich in potassium, such as the DASH diet, and may lead to hyperkalemia in CKD. The guideline recognizes observational data showing $\mathrm{J}$-shaped relationships of urine sodium excretion (a proxy for dietary salt intake) with clinical outcomes, and felt this was an unsettled issue whether there is an unsafe lower limit of salt intake, in other words whether the relationship between salt intake and outcomes is linear or J-shaped.

Regular exercise reduces BP in the general population but the evidence in CKD is limited. As the guideline states, nonetheless, the systematic review of the guideline in the CKD population found low-quality evidence suggesting that physical activity decreases BP and body weight and improves quality of life. Observational data also show a dose-response relationship between greater levels of physical activity and lower risk of mortality in CKD patients. Therefore, the guideline suggests moderate-intensity regular exercise for a cumulative duration of at least $150 \mathrm{~min}$ per week for patients with high BP and CKD, depending on the level of cardiovascular and physical fitness, frailty, or cognitive impairment. The recent KDIGO 2020 Guideline for Diabetes Management in CKD makes similar recommendations; however, for BP in CKD, there is less evidence than for the management of diabetes.

\section{How Should BP Be Managed in CKD Patients, With and Without Diabetes, Not Receiving Dialysis? (Chapter 3)}

\section{Target Blood Pressure}

Potentially the most debated recommendation of the guideline suggests that adults with high $\mathrm{BP}$ and $\mathrm{CKD}$ not receiving dialysis should be treated to a target SBP of $<120 \mathrm{~mm} \mathrm{Hg}$, if tolerated, with the indispensable prerequisite that standardized office measurement technique be employed (Table 2). This recommendation is not applicable to patients receiving dialysis, those with a kidney transplant, or to children less than 18 years old. In the GRADE system, this recommendation is classified as weak. In particular, there is limited evidence for the recommendation in specific subgroups of patients, for example, in those with eGFR $<30 \mathrm{~mL} / \mathrm{min} / 1.73 \mathrm{~m}^{2}$, with diabetes, aged $>90$ years old and $<50$ years old, the very frail, with very low diastolic BP (DBP), or with "white coat" or severe hypertension.

This SBP target is lower than the BP $<130 / 80 \mathrm{~mm} \mathrm{Hg}$ target recommended in the KDIGO $2012 \mathrm{BP}$ guideline. It is also lower than recommended targets for $\mathrm{CKD}$ by $\mathrm{ACC} /$ $\mathrm{AHA}^{2}$ and ESC/ESH [8]; the former recommends SBP
$<130 \mathrm{~mm} \mathrm{Hg}$ while the latter recommends SBP 130 $140 \mathrm{~mm} \mathrm{Hg}$ for CKD. The SBP target of $<120 \mathrm{~mm} \mathrm{Hg}$ in the current KDIGO guideline is, however, consistent with both Hypertension Canada [9] and National Heart Foundation of Australia guidelines.

This recommendation was controversial based on the responses received from the guideline public review. We hope, however, that further clarification of the data used in making this recommendation and the rationale provided in the final guideline document render this recommendation more convincing.

\section{What Were the Main Issues Brought Up During the Public Review?}

In the following, we will list those main issues as subheadings and discuss them further.

\section{Practicality of Standardized Office BP Measurement}

While the necessity to measure BP rigorously was agreeable to most, several reviewers opined that standardized BP measurements are not practical in many clinical settings. It was also erroneously assumed that in SPRINT and ACCORD only unattended (i.e., in the absence of a healthcare provider) standardized BP measurements were performed when neither trial stipulated unattended versus attended BP measurements in its protocol. While an automated device was used in SPRINT and ACCORD, standardized BP readings depend on the appropriate setting and not on the BP device (Table 1). Some automated devices are costly but it lessens the clinic burden by allowing healthcare workers to perform other tasks while BP measurements are taken.

Routine or casual BP measurement without proper preparations may be more convenient, but it has not been used in any large RCT and has an unpredictable relationship to standardized BP in an individual with CKD [3]. The argument of practicality over accuracy is not applicable to other aspects of clinical medicine (e.g., would one use a "casual" potassium measurement with a standard error of $0.5 \mathrm{mmol} / 1$ ?) and the Work Group believed likewise this should not be applicable to BP management.

\section{Limited Evidence Based on a Single Study}

As the guideline states, the recommendation of an SBP target of $<120 \mathrm{~mm} \mathrm{Hg}$, measured with standard office technique, is weak by GRADE standards (Grade $2 B$ ) because it is based on a single, albeit high-quality, RCT with a predefined $\mathrm{CKD}$ subgroup [10]. Indeed, SPRINT included a predefined analysis of its CKD subgroup demonstrating improved cardiovascular and survival benefits and potentially cognitive benefits 
with an SBP target of $<120 \mathrm{~mm} \mathrm{Hg}$, compared to $<140 \mathrm{~mm} \mathrm{Hg}$. While this is the only large outcome trial demonstrating benefits for this low SBP target, the trial execution and results are strong. In addition, secondary analysis of the ACCORD trial showed similar cardiovascular benefits with a SBP target of $<120 \mathrm{~mm} \mathrm{Hg}$ in the subgroup with standard glycemic control, consistent with SPRINT, although the CKD subset in ACCORD was small.

There are three other trials testing different BP targets in CKD published at the time of the original KDIGO 2012 BP guideline. These three trials were powered for GFR-based outcomes, but had accumulated only few events for other clinical outcomes such as mortality or cardiovascular events. Two of these trials, MDRD [11] and AASK [12], used targets based on mean arterial pressure (MAP) and not SBP. The overwhelming majority of non-CKD outcome trials testing different BP targets or comparing different antihypertensive drugs did not use MAP. MAP is also not used clinically in adults. The third trial, REIN-2 [13], did not test a SBP target as low as SPRINT and ACCORD. Hence, SPRINT is the only trial that provides valuable guidance on the SBP target recommended in the current guideline.

While the KDIGO 2021 guideline recommends a single SBP target in CKD, we clearly emphasize individualization of BP management, including consideration of patient characteristics, tolerability, preferences, and life expectancy based on other comorbidities, which are all crucial factors in medical management.

\section{Older Adult Population May Not Benefit from Lower SBP}

Older, frailer adults frequently present with high BP and CKD. One may surmise that the risk-benefit ratio is less favorable with a SBP $<120 \mathrm{~mm} \mathrm{Hg}$ target in these individuals. However, the findings from SPRINT do not support this notion. In fact, absolute cardiovascular and survival benefit of the SBP $<120 \mathrm{~mm} \mathrm{Hg}$ target, compared to $\mathrm{SBP}<140 \mathrm{~mm} \mathrm{Hg}$, was also seen in older adults, including those with CKD, and in the frail subpopulation. Even the risk of developing cognitive impairment appeared to be lower with a target SBP $<120$ $\mathrm{mm} \mathrm{Hg}$. Adverse event rates in the older adult subpopulation of SPRINT were also quite low. There was no significant increase in severe adverse events, such as orthostatic hypotension, syncope, or injurious falls, in the intensive treatment group. It should be noted, however, that SPRINT did not include people residing in nursing homes; the risk-benefit ratio in this subpopulation is therefore less certain.

\section{Subpopulations in CKD}

SPRINT did not include all stages of CKD. In particular, patients with eGFR $<20 \mathrm{~mL} / \mathrm{min} / 1.73 \mathrm{~m}^{2}$ or proteinuria $>1$ $\mathrm{g} / \mathrm{g}$ were explicitly excluded. There were, in fact, few patients with eGFR $<30 \mathrm{~mL} / \mathrm{min} / 1.73 \mathrm{~m}^{2}$ enrolled. There is, however, no substantial evidence to suggest that these CKD subgroups would fare differently with the recommended SBP target of $<120 \mathrm{~mm} \mathrm{Hg}$ recommended by KDIGO than the CKD subgroups that were well represented in SPRINT. Trials in adults with polycystic kidney disease also support the beneficial effects of a low target BP of 95/60 to $110 / 75 \mathrm{~mm} \mathrm{Hg}$.

The issue of target BP in diabetes with $\mathrm{CKD}$ has not been clearly resolved. SPRINT excluded participants with a history of diabetes, but the subgroup with impaired glucose metabolism (fasting glucose $>100 \mathrm{mg} / \mathrm{dL}$ ) exhibited similar cardiovascular benefits as in those with normal fasting glucose levels [14•]. In contrast, ACCORD studied exclusively people with diabetes and randomized participants to the same SBP targets measured by the same technique (standardized AOBP) as in SPRINT (<120 mm Hg vs. $<140 \mathrm{~mm} \mathrm{Hg}$ ) [15]. However, ACCORD randomized few people with eGFR $<60 \mathrm{~mL} / \mathrm{min} /$ $1.73 \mathrm{~m}^{2}$. ACCORD showed no benefit on the primary composite cardiovascular outcome but a substantial reduction in stroke risk with the lower BP target was observed. Post hoc analyses of the ACCORD subgroups defined by its factorial study design suggested cardiovascular benefit of the lower BP target in those randomized to standard glycemic control [16•]. Based on these and other data, the current guideline Work Group felt that patients with concomitant diabetes and CKD are also likely to derive cardiovascular benefits from a target SBP of $<120 \mathrm{~mm} \mathrm{Hg}$. Given the remaining uncertainty and the growing large population of people with concomitant diabetes and CKD, a large definitive RCT in this population is warranted.

\section{Is Intense SBP Lowering Renoprotective?}

BP lowering has traditionally been considered to be a renoprotective strategy. The three $\mathrm{CKD}$ trials mentioned above, MDRD [11], AASK [12], and REIN-2 [13], were designed to examine kidney outcomes, comparing two BP targets. As noted, MDRD and AASK targeted MAP, instead of SBP or DBP, with a lower MAP target of $<92 \mathrm{~mm} \mathrm{Hg}$ (for example, $125 / 75 \mathrm{~mm} \mathrm{Hg}, 160 / 58 \mathrm{~mm} \mathrm{Hg}$, or other combinations) and the target also varied by age. Results related to kidney outcomes during the trial, including changes in GFR, were inconclusive with a potential effect modification by baseline proteinuria. In apparent contrast to MDRD and AASK, the SBP target of $<120 \mathrm{~mm} \mathrm{Hg}$ caused a slightly but statistically significantly faster, rather than slower, decline of eGFR in the CKD subgroup in SPRINT. Similar results were found in the ACCORD [15] and SPS3 [17] cohorts. Yet urine albumin was lower with the lower BP target in the CKD subgroup in SPRINT; lower albuminuria is usually associated with more favorable long-term kidney outcomes. It is, therefore, unsettled whether lowering SBP from $<140$ to $<120 \mathrm{~mm}$ 
Hg would prevent or hasten kidney failure in the long term and whether baseline proteinuria is an effect modifier with intense SBP lowering.

\section{Other BP Guidelines Proffer Different Targets}

Some, but not all guidelines, from other organizations recommend $\mathrm{BP}$ targets that are different from the current KDIGO 2021 Guideline for the Management of BP in CKD. As mentioned previously, Hypertension Canada also recommends a SBP target of $<120 \mathrm{~mm} \mathrm{Hg}$, but the 2017 ACC/AHA guideline offers a target of $<130 /<80 \mathrm{~mm} \mathrm{Hg}$ for patients with CKD. This disagreement, based on the same available evidence, may be puzzling, but is a common feature of scientific discourse. The ESC/ESH guideline recommends a SBP target of 130-139 $\mathrm{mm} \mathrm{Hg}$ [8], while the UK National Institute of Health and Care Excellence (NICE) guideline recommends a SBP target of 120-139 mm Hg for CKD [18].

One of the reasons for this variability may be related to the concern by some that clinicians might apply the target based on routine, rather than standardized, office BP readings, potentially resulting in overtreatment in many and undertreatment in some patients. While the KDIGO Work Group also shares this concern, the guideline panel believed that patients should not be penalized for suboptimal clinical practice; standardized office BP should be used to guide therapy so that a SBP $<120 \mathrm{~mm} \mathrm{Hg}$ can be reasonably targeted. For these reasons, the KDIGO 2021 BP Guideline recommends the use of standardized office BP measurements, to avoid both under- or overtreatment when targeting SBP $<120 \mathrm{~mm} \mathrm{Hg}$, and to provide evidence-based clinical treatment to CKD patients with high BP.

\section{Antihypertensive Drugs}

The KDIGO Work Group acknowledges that in those with high BP and CKD, there are RCTs testing outcomes for renin-angiotensin system inhibitors (RASi), calcium channel blockers (CCB), and beta-blockers. Those drugs have been compared to placebo as well as to each other. There are no such RCTs with combination therapies of antihypertensive drugs or other drugs beyond RASi, CCB, and beta-blockers.
Fig. 1 Treatment algorithm for intensive systolic blood pressure arm (target $\mathrm{SBP}<120 \mathrm{~mm} \mathrm{Hg}$ ) in SPRINT trial protocol

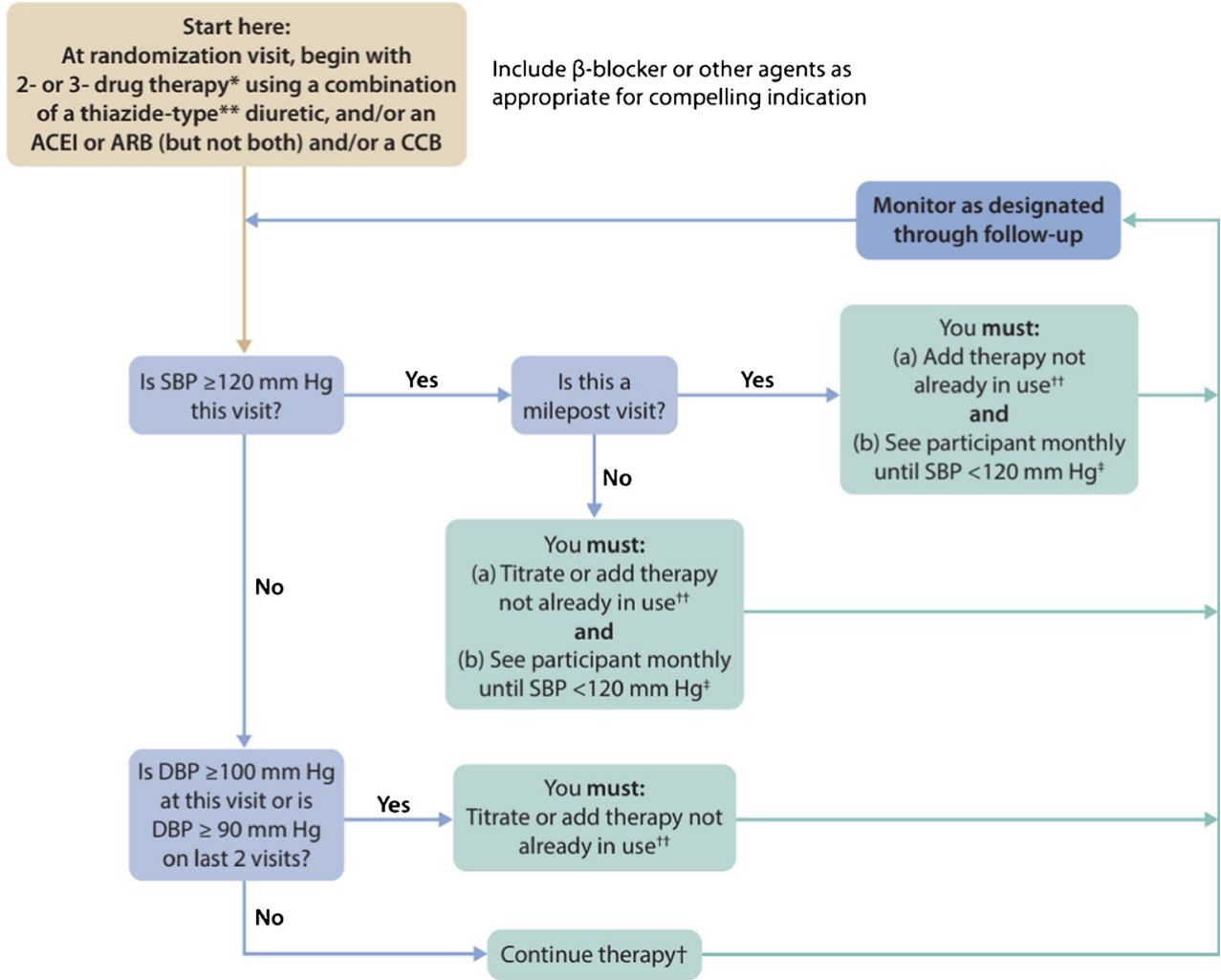

${ }^{*}$ May begin with a single agent for participants 75 years old or older with SBP $<140 \mathrm{~mm} \mathrm{Hg}$ on $0-1$ medications at study entry. A second medication should be added at the 1 month visit if participant is asymptomatic and SBP $\geq 130 \mathrm{~mm} \mathrm{Hg}$. ** May use loop diuretic for participants with advanced CKD.

Unless side effects warrant change in therapy.

${ }^{\dagger}$ Consider adding a fifth anti-hypertensive medication.

*Or until clinical decision is made that therapy should not be increased further.

(From: SPRINT Research Group, et al. N Engl J Med 2015; 373: 2103-2116. Copyright @ 2015 Massachusetts Medical Society. Reprinted with permission from Massachusetts Medical Society) [10]. 
Any treatment advice regarding antihypertensive drugs in $\mathrm{CKD}$, beyond monotherapy, is based on mechanisms of action or pharmacology of the drugs, extrapolations from trials in primary hypertension in the non-CKD population, and expert opinions. As an example, Fig. 1 displays the treatment algorithm used in the SPRINT trial [10].

Despite the shortcomings noted above, those with high $\mathrm{BP}$ and CKD should receive a combination of two or more drugs when $\mathrm{BP}$ is $\geq 20 \mathrm{~mm} \mathrm{Hg}$ above target, as recommended by the current guideline. This advice is similar to the suggestions from other guidelines. We recognize that starting with two rather than a single drug likely elicits a greater drop in BP and that there are no data on clinical outcomes using this approach. The current guideline further cites a recent network meta-analysis of Xie et al. which included 119 RCTs with approximately 65,000 participants with or without diabetes and with or without albuminuria [19]. In those with CKD, treatment with RASi, as compared to placebo or other antihypertensive drugs, reduced the risk for kidney failure and of major cardiovascular events. In addition, ACEi, but not ARB, reduced the odds of death. In the particular subgroup with concomitant diabetes and albuminuric CKD, a meta-analysis by the KDIGO Guideline Evidence Review Team found RASi decreases the risk for doubling of serum creatinine but not mortality, with no differences between ACEi and ARB. These subgroup data must be interpreted with caution because of limited statistical power.

Diuretics are standard drugs in the treatment of high BP in CKD. Sodium retention and a positive fluid balance are thought to contribute substantially to high BP in CKD. There are, however, no solid RCT data on diuretics for this indication, at least not for thiazides or thiazide-like drugs. Very recently, an RCT with a diuretic, the mineralocorticoid receptor antagonist (MRA) finerenone, demonstrated kidney and cardiovascular benefits in those with diabetic CKD. Those data could not be included in the evidence review because its publication coincided with the submission of our guideline.

\section{Conclusions}

This paper summarized the major aspects of the new KDIGO 2021 guideline on the management of high BP in CKD. We did not discuss the chapters on kidney transplant recipients and on children with CKD since the latter groups of patients are usually followed up by specialized physicians. Herein we stress the importance of correct, that is standardized office measurement of BP. It is on the background of that measurement, which was used in essentially all RCTs examining target BP, that we recommend a target $\mathrm{BP}$ of $<120 \mathrm{~mm}$ $\mathrm{Hg}$ systolic in all people with CKD. Although the strength of evidence for that recommendation varies depending on specific subgroups or etiologies of $\mathrm{CKD}$ this again reiterates the importance for individualization of BP management based on patient preferences and carefully assessing the risk profile and weighing the benefits and harms.

Acknowledgements A special debt of gratitude is owed to the following colleagues for their contributions to this important guideline effort: Melissa Thompson, Debbie Maizels, Suetonia C. Palmer, Giovanni F.M. Strippoli, Fiona Russell, Gail Y, Higgins, Tess E. Cooper, Nicole Evangelidis, Brydee Cashmore, Rabia Khalid, Claris Teng, Elisabeth Hodson, Emma McMahon, Patrizia Natale, Narelle Willis, Michel Jadoul, Wolfgang Winkelmayer, Kathleen Conn, Danielle Green, Tanya Green, and John Davis.

Funding KDIGO. No funding is accepted from external parties for the development of specific guidelines.

Data Availability All data are available at https://kdigo.org/guidelines/ blood-pressure-in-ckd/.

\section{Declarations}

Conflict of Interest Tara I. Chang, MD, MS: consultancy: Janssen; Novo Nordisk; grants/grants pending: Satellite Health Care*, National Institutes of Health, American Heart Association; workshop honoraria: Fresenius.

Alfred K. Cheung, MD: consultancy: Boehringer Ingelheim; grants/ grants pending: National Institutes of Health for SPRINT.

William C. Cushman, MD: consultancy: Sanofi; grants/grants pending: National Institute on Aging*; National Institutes of Health*; and Eli Lilly*.

Susan L. Furth, MD, PhD: grants/grants pending: National Institutes of Health*.

Joachim H. Ix, MD, MAS: grants/grants pending: Baxter International*; National Institutes of Diabetes and Digestive and Kidney Diseases*; and American Heart Association*; travel expenses: American Society of Nephrology and Institute of Medicine.

Fan Fan Hou, MD, PhD: consultancy: AbbVie and AstraZeneca; grants/grants pending: National Natural Science Foundation of China; Ministry of Science and Technology of China.

Gregory A. Knoll, MD, MSc, FRCPC: grants/grants pending: Canadian Institutes of Health Research*.

Johannes F.E. Mann, MD: consultancy: AstraZeneca; Bayer Healthcare; Boehringer Ingelheim; Fresenius Medical Care; Novo Nordisk; and Vifor Pharma; grants/grants pending: AbbVie; Boehringer Ingelheim; Canadian Institutes Health Research; Celgene; European Union; Idorsia; Novo Nordisk; Roche; Sanofi; and Sandoz; Speaker Bureaus: Amgen; AstraZeneca; Braun; Fresenius Medical Care; Gambro; Medice; Novo Nordisk; Roche.

Paul Muntner, PhD MHS, FASH, FAHA: none

Roberto Pecoits-Filho, MD, PhD, FASN, FACP: consultancy: AstraZeneca; Akebia; Fresenius Medical Care; and Novo Nordisk; grants/grants pending: Fresenius Medical Care*; Speaker Bureaus: AstraZeneca and Novo Nordisk.

Mark J. Sarnak, MD, MS: consultancy: Akebia*; grants/grants pending: National Institutes of Health*.

Sheldon W. Tobe, MD, MScCH (HPTE): honorarium: CHEP+ a physician organization CPD not-for-profit organization that provides Canadian accredited continuing education. CHEP+ has received support in accordance with accreditation protocols from The University of Toronto, The Royal College of Physicians and Surgeons of Canada, Astra Zeneca, Bayer, Bristol Myers Squibb, Janssen, Pfizer. 
Charles R.V. Tomson, DM, FRCP: travel: Attendance at a London Hatter Institute meeting organized by the Lancet and University College London with arm's- length funding from pharmaceutical companies.

The other authors declare that they have no conflict of interest.

* monies to institution

Human and Animal Rights and Informed Consent This article does not contain any studies with human or animal subjects performed by any of the authors.

\section{References}

Papers of particular interest, published recently, have been highlighted as:

- Of importance

•- Of major importance

1.• Kidney Disease: Improving Global Outcomes Blood Pressure Work Group. KDIGO 2021 Clinical Practice Guideline for Management of Blood Pressure in Chronic Kidney Disease. Kidney Int. 2021;99:S1-S87. This guideline updates the previous 2012 edition and is accompanied by an Executive Summary which offers a quick overview of the key takeaways from this guidance document.

2. Whelton PK, Carey RM, Aronow WS, et al. 2017 ACC/AHA/ AAPA/ABC/ACPM/AGS/APhA/ASH/ASPC/NMA/PCNA guideline for the prevention, detection, evaluation, and management of high blood pressure in adults: a report of the American College of Cardiology/American Heart Association Task Force on Clinical Practice Guidelines. J Am Coll Cardiol. 2018;71: e127-248.

3.• Agarwal R. Implications of blood pressure measurement technique for implementation of Systolic Blood Pressure Intervention Trial (SPRINT). J Am Heart Assoc. 2017;6:e004536. This key paper demonstrated that in individuals with CKD, there is a wide variability in terms of BP measured "routinely" vs. by standardized technique, and that these differences are unpredictable in any given individual.

4. Johnson KC, Whelton PK, Cushman WC, et al. Blood pressure measurement in SPRINT (Systolic Blood Pressure Intervention Trial). Hypertension. 2018;71:848-57.

5. Uhlig K, Patel K, Ip S, et al. Self-measured blood pressure monitoring in the management of hypertension: a systematic review and meta-analysis. Ann Intern Med. 2013;159:185-94. This report provides a detailed analysis of the main 3 trials up to 2012 that examined target $\mathrm{BP}$ in people with high $\mathrm{BP}$ and $\mathrm{CKD}$, with kidney disease progression as main outcome. The arrival of SPRINT-CKD trial after this publication has furnished additional CV outcome data which provided the impetus for this guideline update.

6. World Health Organization. Guideline: Sodium Intake for Adults and Children. Available at: https://apps.who.int/iris/bitstream/ handle/10665/77985/9789241504836_eng.pdf?sequence $1 / 41$; 2012. Accessed January 15, 2021.

7. Kidney Disease: Improving Global Outcomes Diabetes Work Group. KDIGO 2020 Clinical Practice Guideline for Diabetes
Management in Chronic Kidney Disease. Kidney Int. 2020;98: S1-S115.

8. Williams B, Mancia G, Spiering W, et al. 2018 ESC/ESH Guidelines for the management of arterial hypertension: the Task Force for the management of arterial hypertension of the European Society of Cardiology and the European Society of Hypertension: the Task Force for the management of arterial hypertension of the European Society of Cardiology and the European Society of Hypertension. J Hypertens. 2018;36:1953-2041.

9. Rabi DM, McBrien KA, Sapir-Pichhadze R, et al. Hypertension Canada's 2020 Comprehensive Guidelines for the prevention, diagnosis, risk assessment, and treatment of hypertension in adults and children. Can J Cardiol. 2020;36:596-624.

10. SPRINT Research Group, Wright JT Jr, Williamson JD, et al. A randomized trial of intensive versus standard blood-pressure control. N Engl J Med. 2015;373:2103-16.

11. Klahr S, Levey AS, Beck GJ, et al. The effects of dietary protein restriction and blood-pressure control on the progression of chronic renal disease. Modification of Diet in Renal Disease Study Group. N Engl J Med. 1994;330:877-84.

12. Wright JT Jr, Bakris G, Greene T, et al. Effect of blood pressure lowering and antihypertensive drug class on progression of hypertensive kidney disease: results from the AASK trial. JAMA. 2002;288:2421-31.

13. Ruggenenti P, Perna A, Loriga G, et al. Blood-pressure control for renoprotection in patients with non-diabetic chronic renal disease (REIN-2): multicentre, randomised controlled trial. Lancet. 2005;365:939-46.

14. Bress AP, King JB, Kreider KE, et al. Effect of intensive versus standard blood pressure treatment according to baseline prediabetes status: a post hoc analysis of a randomized trial. Diabetes Care. 2017;40:1401-8. The results from this SPRINT post hoc analysis demonstrated similar benefits in those with intensive SBP treatment among those with prediabetes and fasting normoglycemia.

15. ACCORD Study Group, Cushman WC, Evans GW, et al. Effects of intensive blood-pressure control in type 2 diabetes mellitus. N Engl J Med. 2010;362:1575-85.

16. Beddhu S, Chertow GM, Greene T, et al. Effects of intensive systolic blood pressure lowering on cardiovascular events and mortality in patients with type 2 diabetes mellitus on standard glycemic control and in those without diabetes mellitus: reconciling results from ACCORD BP and SPRINT. J Am Heart Assoc. 2018;7:e009326 The results of intensive SBP control on cardiovascular disease events and all-cause mortality appear to vary depending on the intensity of glycemic control.

17. S.P.S. Study Group, Benavente OR, Coffey CS, et al. Bloodpressure targets in patients with recent lacunar stroke: the SPS3 randomised trial. Lancet. 2013;382:507-15.

18. National Institute for Health and Care Excellence: hypertension in adults: diagnosis and management. NICE guideline [NG136]. Available at: https://www.nice.org.uk/guidance/ng136; 2019. Accessed January 15, 2021.

19. Xie X, Liu Y, Perkovic V, et al. Renin-angiotensin system inhibitors and kidney and cardiovascular outcomes in patients with CKD: a Bayesian network meta-analysis of randomized clinical trials. Am J Kidney Dis. 2016;67:728-41.

Publisher's Note Springer Nature remains neutral with regard to jurisdictional claims in published maps and institutional affiliations. 